\title{
(2) OPEN ACCESS
}

\section{Mitochondrial impairment drives intestinal stem cell transition into dysfunctional Paneth cells predicting Crohn's disease recurrence}

\author{
Sevana Khaloian, ${ }^{1}$ Eva Rath ${ }^{0},{ }^{1}$ Nassim Hammoudi, ${ }^{2}$ Elisabeth Gleisinger, ${ }^{1}$ \\ Andreas Blutke, ${ }^{3}$ Pieter Giesbertz, ${ }^{4}$ Emanuel Berger, ${ }^{1}$ Amira Metwaly, ${ }^{1}$ \\ Nadine Waldschmitt, ${ }^{1}$ Matthieu Allez (1) ${ }^{2}$ Dirk Haller (1) ${ }^{1,5}$
}

- Additional material is published online only. To view please visit the journal online (http://dx.doi.org/10.1136/ gutjn-2019-319514)

For numbered affiliations see end of article.

\section{Correspondence to}

Professor Dirk Haller, Chair of Nutrition and Immunology, Technische Universität

München, München, Bayern 85350, Germany;

dirk.haller@tum.de

SK and ER contributed equally.

Received 20 July 2019 Revised 23 January 2020 Accepted 3 February 2020 Published Online First 28 February 2020

\section{Linked}

- http://dx.doi.org/10.1136/ gutjnl-2019-319523

Check for updates

(C) Author(s) (or their employer(s)) 2020. Re-use permitted under CC BY-NC. No commercial re-use. See rights and permissions. Published by BMJ.

To cite: Khaloian S, Rath $\mathrm{E}$, Hammoudi N, et al. Gut 2020;69:1939-1951.

\section{ABSTRACT}

Objective Reduced Paneth cell (PC) numbers are observed in inflammatory bowel diseases and impaired PC function contributes to the ileal pathogenesis of Crohn's disease (CD). PCs reside in proximity to $\mathrm{Lgr}^{+}$ intestinal stem cells (ISC) and mitochondria are critical for ISC-renewal and differentiation. Here, we characterise ISC and PC appearance under inflammatory conditions and describe the role of mitochondrial function for ISC niche-maintenance.

Design Ileal tissue samples from patients with $C D$, mouse models for mitochondrial dysfunction $\left(\mathrm{Hsp} \mathrm{O}^{\Delta /}\right.$ $\left.{ }^{\triangle I S C}\right)$ and $C D$-like ileitis (TNF $\left.{ }^{\triangle A R E}\right)$, and intestinal organoids were used to characterise PCs and ISCs in relation to mitochondrial function.

Results In patients with CD and TNF ${ }^{\triangle A R E}$ mice, inflammation correlated with reduced numbers of Lysozyme-positive granules in PCs and decreased Lgr5 expression in crypt regions. Disease-associated changes in PC and ISC appearance persisted in non-inflamed tissue regions of patients with $C D$ and predicted the risk of disease recurrence after surgical resection. ISCspecific deletion of Hsp60 and inhibition of mitochondrial respiration linked mitochondrial function to the aberrant PC phenotype. Consistent with reduced stemness in vivo, crypts from inflamed TNF ${ }^{\triangle A R E}$ mice fail to grow into organoids ex vivo. Dichloroacetate-mediated inhibition of glycolysis, forcing cells to shift to mitochondrial respiration, improved ISC niche function and rescued the ability of TNF ${ }^{\triangle A R E}$ mice-derived crypts to form organoids. Conclusion We provide evidence that inflammationassociated mitochondrial dysfunction in the intestinal epithelium triggers a metabolic imbalance, causing reduced stemness and acquisition of a dysfunctional PC phenotype. Blocking glycolysis might be a novel drug target to antagonise PC dysfunction in the pathogenesis of $C D$.

\section{INTRODUCTION}

Crohn's disease (CD), belonging to the group of inflammatory bowel diseases (IBD), is characterised by transmural acute and chronic inflammation of intestinal tissue regions, typically involving the terminal ileum. ${ }^{1}$ In the pathogenesis of $\mathrm{CD}$, multiple genetic risk factors together with environmental triggers result in a disturbed immune response

\section{Significance of this study}

What is already known on this subject?

- Reduced Paneth cell (PC) numbers are frequently observed in inflammatory bowel diseases (IBD) and impaired PC function is a feature of ileal Crohn's disease (CD).

- PCs are involved in mucosal defence and support the intestinal stem cell (ISC) niche.

- Mitochondrial dysfunction and alterations in energy metabolism in general are implicated during the onset and the course of IBD.

- Mitochondrial function, metabolism and mitochondrial unfolded protein response are involved in intestinal epithelial cell differentiation and determine the cellular phenotype.

What are the new findings?

- Reduced expression of the ISC marker Lgr5, in addition to reduced PC granularity, correlates with inflammation in patients with $C D$ and $C D$ like TNF $^{\triangle A R E}$ mice.

- Morphological appearance of PCs and ISC in non-affected tissue margins predicts early postoperative endoscopic recurrence in patients with $\mathrm{CD}$.

- Induction of mitochondrial dysfunction in ISC by Hsp60 loss results in overall reduced Lgr5 expression and causes differentiation of $\mathrm{Lgr}^{+}$ into aberrant PCs.

- Reinforcing mitochondrial respiration by inhibition of glycolysis restores inflammationimprinted dysfunction of the ISC niche.

How might it impact on clinical practice in the foreseeable future?

- We provide evidence that impaired mitochondrial function is linked to the CD-associated loss of stemness and the generation of dysfunctional PC phenotypes. Demonstrating a proof-of-concept for targeting ISC alterations by implementing a drug-related metabolic shift, we rationalise a novel treatment approach for CD.

towards a dysbiotic commensal microbiota. ${ }^{2}$ The intestinal epithelium as interface between microbiota and host critically contributes to intestinal homeostasis, and alterations in intestinal epithelial 
cell (IEC) subtypes including reduced numbers of goblet cells and Paneth cells (PCs) are frequently observed under inflammatory conditions. ${ }^{3}$ PCs are located in the crypt base of the small intestine, residing between Leucine-rich repeat-containing G-protein coupled receptor (Lgr) 5 crypt base columnar (CBC) intestinal stem cells (ISCs) and via secretion of antimicrobial peptides (AMPs) such as lysozyme, defensins (cryptdins), angiogenin-4 (Ang4) and secretory phospholipase A2, PCs contribute to pathogen clearance and shape the commensal microbiota. ${ }^{45}$

In addition to mucosal defence, PCs provide essential signals for maintenance of the ISC niche. Among those are Notch ligand (Dll4), and secreted factors like EGF and Wnt $3,{ }^{6}$ and also more recently identified metabolic signalling molecules including cyclic ADP ribose (cADPR) ${ }^{7}$ and lactate, ${ }^{8}$ facilitating optimal stem cell function. Moreover, on acute injury, PCs themselves serve as a reserve stem cell population, restoring $\mathrm{Lgr} 5^{+}$ISC via dedifferentiation, thereby contributing to tissue regeneration. ${ }^{9-11}$

Genetic risk variants of prominent CD-relevant genes involved in autophagy (ATG16L1), bacterial-sensing (NOD2), endoplasmic reticulum (ER) stress response (XBP1) and Wnt signalling (TCF4) were shown to affect PC function in terms of AMP production and secretion, and loss of PC defensins is particularly associated with ileal phenotypes of CD. ${ }^{32-16}$ Yet, it is controversial whether ileal CD is a specific disorder of PCs, or if loss of PC function is merely associated, but not causal for the development of CD. IEC-specific knockout models developing CD-like ileal inflammation including Caspase $8^{-1 /},{ }^{17}$ Atg $1611^{-1-15}$ and Xbp $1^{-/ 12}$ mice share the loss of PC function as a mechanism of pathogenesis; however, genetic ablation of PCs is not sufficient to generate a CD-associated phenotype. ${ }^{18}{ }^{19} \mathrm{In}$ line with a role of PCs in disease progression, $\mathrm{TNF}^{\mathrm{AARE}}$ mice, a bacterial-driven model of chronic CD-like ileitis, display a loss of lysozyme-positive PCs subsequent to, but not preceding, the onset of TNF-driven tissue pathology. No increase in apoptosis could be observed in crypts from $\mathrm{TNF}^{\mathrm{AARE}}$ mice, suggesting epithelial remodelling rather than PC-specific cell death. ${ }^{20}$ The cause of PC alterations as well as PC fate under chronic inflammatory conditions is currently unknown, though impairment of the exocytosis pathway in PC of mice with hypomorphic expression of the CD susceptibility gene Atg16l1 is paralleled by degenerating mitochondria. ${ }^{14}$ Interestingly, several genetic risk factors affecting mitochondrial function were identified for IBD, ${ }^{21}$ and intestinal inflammation has been suggested as energy-deficiency disease of IECs featuring alterations of the mitochondrial metabolism. ${ }^{22} 23$ Concomitantly, mitochondrial function and signalling pathways like the mitochondrial unfolded protein response (MT-UPR) have emerged as cellular checkpoint of metabolism, stemness and IEC differentiation programme. ${ }^{24}$ We previously demonstrated MT-UPR activation in IEC from patients with IBD and mouse models of intestinal inflammation ${ }^{25}$ and showed that activation of MT-UPR, induced by IEC-specific loss of the mitochondrial chaperone Hsp60, resulted in impaired mitochondrial respiration, and loss of ISC. ${ }^{26}$ However, the cellular origin and specific mechanisms that integrate mitochondrial function into CD pathology remain unknown. The cellular metabolism is increasingly recognised as a determiner of cellular phenotype and stem cell fate, ${ }^{24}$ and taking the essential role of PCs in the regulation of the ISC niche into account, we aimed at characterising PCs and $\mathrm{Lgr} 5^{+}$ISC in the context of mitochondrial function and inflammation.

In this study, we show that reduced PC granularity and reduced Lgr 5 expression correlate with inflammation in patients with CD and $\mathrm{TNF}^{\triangle A R E}$ mice. Importantly, the morphological appearance of the ISC niche in non-affected tissue margins predicts early postoperative endoscopic recurrence in patients with $\mathrm{CD}$, identifying an objective biomarker to select patients for preventive treatment. Induction of mitochondrial dysfunction in ISC by Hsp60 loss results in overall reduced Lgr 5 expression and causes differentiation of $\mathrm{Lgr}^{+}$into aberrant PCs. Remarkably, inhibition of glycolysis is sufficient to override the inflammationimprinted changes of the ISC niche ex vivo and rescued the ability of TNF ${ }^{\triangle \mathrm{ARE}}$ mice-derived crypts to give rise to organoids.

\section{METHODS AND MATERIAL}

All relevant methods and materials can be found in the online supplementary material.

\section{RESULTS}

\section{REDUCED PANETH CELL FUNCTION AND LGR5-EXPRESSION CORRELATE WITH CD-LIKE INFLAMMATION}

In $\mathrm{TNF}^{\triangle \mathrm{ARE}}$ mice, a deletion in the tumour necrosis factor (TNF) AU-rich (adenosin-uracil) elements (ARE) leads to loss of translational control of TNF, resulting in a microbiota-driven transmural inflammation of the distal ileum. ${ }^{20} \mathrm{CD}$-like tissue inflammation gradually develops in $\mathrm{TNF}^{\triangle \mathrm{ARE}}$ mice and has been linked to PC dysfunction. ${ }^{20}$ PCs contain distinct cytoplasmic granules for exocytosis of AMPs, ${ }^{27}$ and lysozyme-positive $\left(\mathrm{Lyz}^{+}\right)$ secretory granule morphology is used as a functional marker for PC phenotype classification. ${ }^{13}{ }^{14}$ We took advantage of the heterogeneity in the grade of CD-like inflammation in $\mathrm{TNF}^{\mathrm{AARE}}$ mice to characterise PCs across a broad range of histopathological scores (HS). Ileal tissue sections derived from $\mathrm{TNF}^{\triangle \mathrm{ARE}}$ mice with no (HS 0), moderate (HS <4) and severe (HS>4) inflammation were stained for Lyz and PCs were subdivided into a highly granular ( $\geq 2 \mathrm{Lyz}^{+}$cytoplasmic granules) and a lowly granular $\left(<2 \mathrm{Lyz}^{+}\right.$cytoplasmic granules and/or diffuse cytoplasmic Lyz staining) phenotype (figure 1A). Representative H\&E stainings of tissue sections at different $\mathrm{HS}$ are given in figure $1 \mathrm{~B}$. Total numbers of $\mathrm{Lyz}^{+}$PCs per crypt (figure 1C) and the percentage of highly granular PCs (figure 1D) inversely correlated with the grade of inflammation and signs of PC abnormalities were already visible in moderately inflamed mice (figure 1C,D). Comparing tissues from non-inflamed $\mathrm{TNF}^{\triangle \mathrm{ARE}}$ mice and wild type (WT) littermates, we observed no differences in the total numbers of PCs. However, non-inflamed TNF ${ }^{\triangle A R E}$ mice showed a mild reduction in $\mathrm{PC}$ granularity (online supplementary figure $1 \mathrm{~A}, \mathrm{~B})$. Confirming reduced $\mathrm{PC}$ function in $\mathrm{TNF}^{\triangle \mathrm{ARE}}$ mice, transcriptional levels of $L y z 1$ and PC-derived AMPs alpha-defensin 5 (Defa5) and Ang4 and ISC-niche supporting Dll4 were reduced (figure 1E). To depict the impact of ileitis-associated changes in PC function on CBC ISC marked by Lgr5 we performed in situ hybridisation for $\operatorname{Lgr} 5$ (figure $1 \mathrm{~F}$ ). The number of dots, representing Lgr5 transcripts, were counted (figure 1G) and crypts were classified as crypts with high $\operatorname{Lgr} 5$ expression ( $\geq 10$ dots) and low Lgr5 expression ( $<10$ dots), respectively. Consistent with reduced PC function, $\operatorname{Lgr} 5^{+}$ISCs were diminished under inflammatory conditions (online supplementary figure 1C) and numbers of crypts with high $\operatorname{Lgr} 5$ expression were inversely correlated with the degree of histopathology (figure $1 \mathrm{H}$ ). Reduced stemness in inflamed $\mathrm{TNF}^{\triangle \mathrm{ARE}}$ mice was confirmed by qPCR analysis of Lgr5 (figure 1I). No differences were observed in crypt morphology and Lgr5 expression between WT and noninflamed $\mathrm{TNF}^{\triangle \mathrm{ARE}}$ mice (online supplementary figure $1 \mathrm{C}, \mathrm{D}$ ). In situ hybridisation for Olfactomedin (Olfm) 4, a broader marker of active ISCs, confirmed reduced stemness under inflammatory conditions (online supplementary figure 1E-G). The 
1A

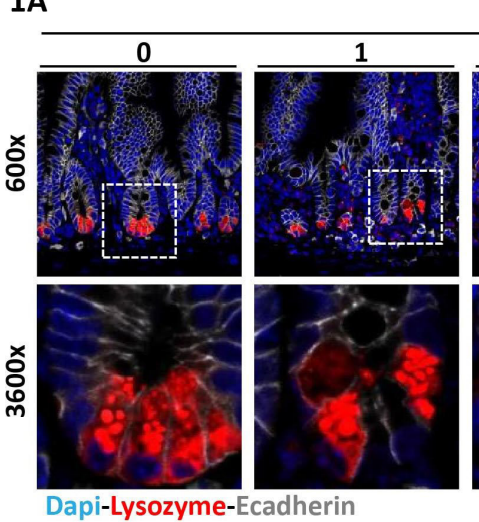

TNF $\triangle$ ARE

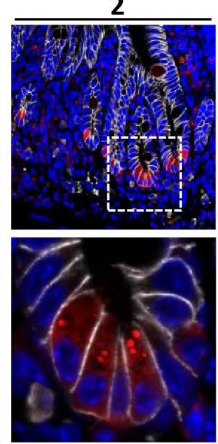

1D
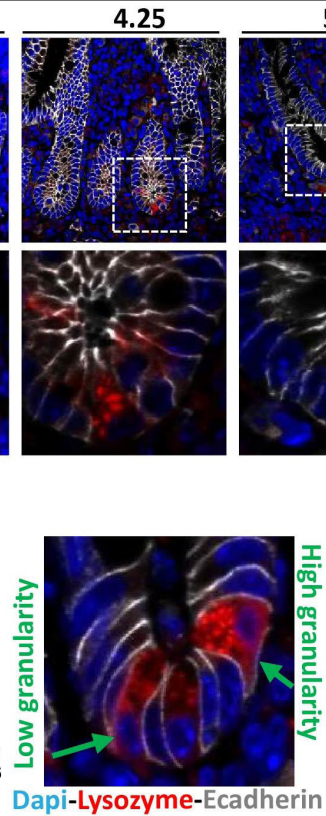

$1 \mathrm{~B}$

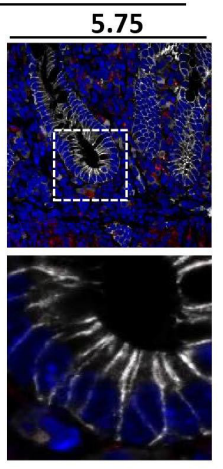

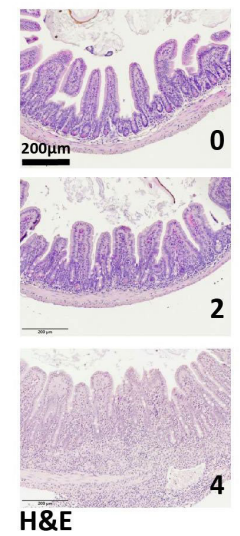

$1 \mathrm{E}$

Figure 1 Paneth cell dysfunction and reduced $L g r 5$-expression correlate with CD-like inflammation in TNF ${ }^{\triangle A R E}$ mice lleal tissue sections from TNF $^{\triangle A R E}$ mice with different levels of inflammation and IEC isolates derived from TNF ${ }^{\triangle A R E}$ mice and WT littermates were analysed. (A) Immunofluorescence (IF) costaining of Lysozyme (red) and E-cadherin (IEC borders, grey) counterstained with Dapi (nuclei, blue), lower panel: higher magnification of the indicated sections. Numbers above the pictures indicate the HS of the respective tissue section. (B) Representative H\&E images for HS 0 (noninflamed), 2 (moderate inflammation), 4 (severe inflammation). (C) Correlation analysis (Pearson) of the number of Lysozyme positive (Lyz ${ }^{+}$) cells and HS and (D) proportions of highly granular Lyz ${ }^{+}$cells ( $\geq 2$ granules) and HS. Right: representative Lyz staining depicting Lyz ${ }^{+}$cells with low and high granularity, respectively. (E) qRT-PCR analysis of IECs for genes involved in PC function $(n=5)$ (F) Representative pictures of $L$ gr5 in situ hybridisation, including magnifications; numbers indicate the respective HS. (G) Illustration of Lgr5 transcript quantification; each dot, indicated by a black arrow represents one Lgr5 transcript. (H) Correlation analysis (Pearson) of the proportion of crypts with high Lgr5 expression ( $\geq 10$ transcripts) and HS. (I) qRT-PCR analysis of IECs for $\operatorname{Lgr} 5(\mathrm{n}=5)$. Statistics were performed by unpaired $t$-test. Bars represent mean+SEM. Asterisks indicate significant differences ${ }^{*} p<0.05,{ }^{* *} p<0.01,{ }^{* * *} p<0.001$. ARE,AU-rich (adenosin-uracil) elements; CD, Crohn's disease; HS, histopathological score; IEC, intestinal epithelial cell; PC, Paneth cell; TNF, tumour necrosis factor; WT, wild type.

parallel decrease of PC function and stemness indicates the tight functional interrelation of the ISC niche under inflammatory conditions.

\section{ABERRANT PC PHENOTYPE AND LGR5-EXPRESSION CORRELATE WITH ACTIVE ILEAL CD}

To verify the relevance of $\mathrm{TNF}^{\mathrm{ARE}}$ mice, we obtained samples from 70 patients with $C D$ undergoing surgical resection from a previously published prospective study. ${ }^{28}$ Early postoperative lesions observed by ileocolonoscopy best predict the course of disease and ileocolonoscopy is currently the gold standard to assess the risk of clinical recurrence. ${ }^{29}$ Ileal tissue margins were collected during resection surgery and a postoperative endoscopy was performed 6-12 months later to assess the endoscopic recurrence according to the Rutgeerts score. Endoscopic recurrence was defined as a Rutgeerts score $\geq i 2$. Classification of patients with $\mathrm{CD}$ according to the presence or absence of inflammation in the resected tissue samples at baseline, and classification of postoperative endoscopic recurrence, is schematically shown in figure $2 \mathrm{~A}$, and patients' characteristics at the time of surgery are compiled in online supplementary table 1. Consistent with TNF ${ }^{\triangle A R E}$ mice, numbers of $\mathrm{LYZ}^{+}$PCs and highly granular PCs ( $\geq 2 \mathrm{LYZ}^{+}$cytoplasmic granules) were significantly reduced in tissue margins classified as inflamed at time of surgery 
2A

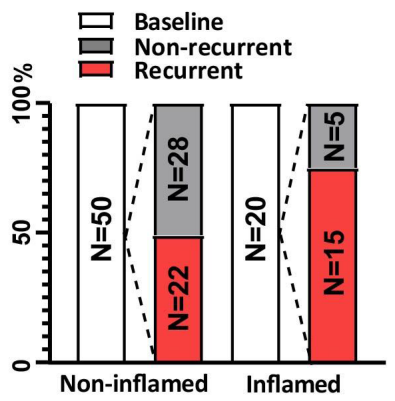

2C
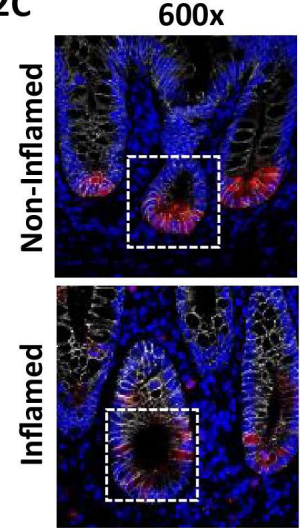

Dapi-Lysozyme-Ecadherin
2B

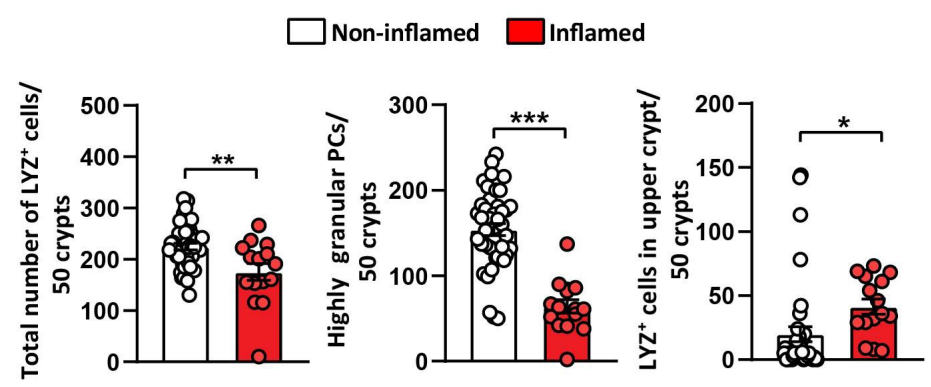

2D

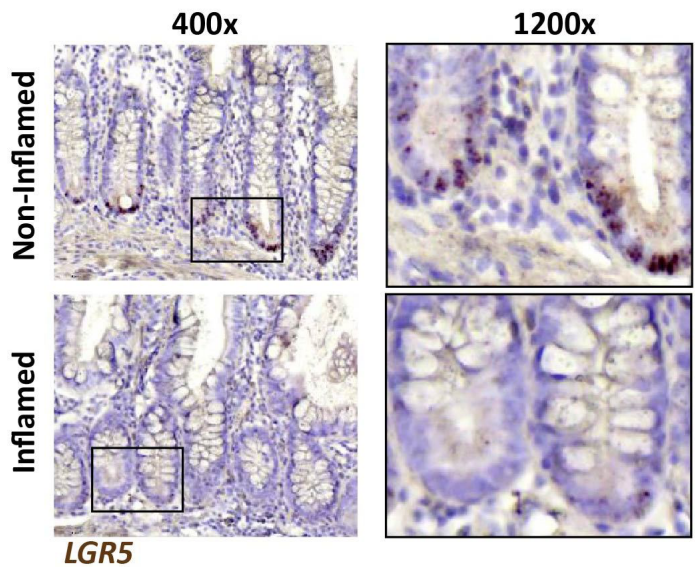

2E
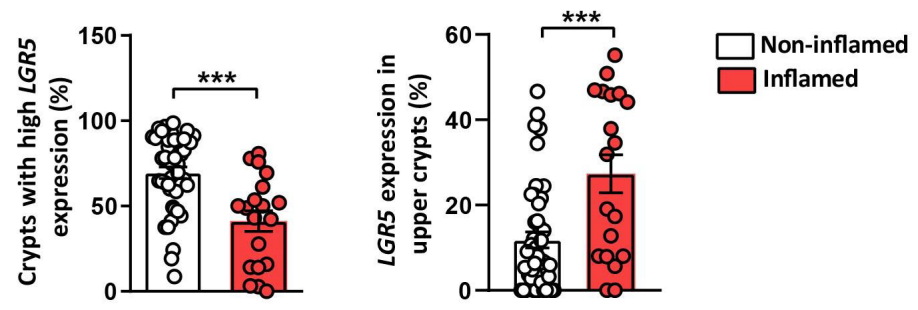

Figure 2 Aberrant Paneth cell phenotype and LGR5-expression correlate with disease activity in ileal tissue margins of patients with CD lleal tissue sections of patients with $C D$ undergoing resection surgery were analysed and tissue margins classified as non-inflamed and inflamed, respectively, at time of surgery were compared. (A) Overview of CD patient numbers for baseline and endoscopic follow-up disease classification. (B) Quantification of the total number of $\mathrm{LYZ}^{+}$cells, highly granular $\mathrm{LYZ}^{+}$cells ( $\geq 2$ granules) and number of $\mathrm{LYZ}^{+}$cells in upper crypt based on LYZ IF staining. (C) Representative pictures of IF costaining of Lysozyme (red) and E-cadherin (IEC borders, grey) counterstained with Dapi (nuclei, blue), right panel: higher magnification of the indicated sections. (D) Representative pictures of LGR5 in situ hybridisation, including magnifications. (E) Quantification of $L G R 5$ in situ hybridisation giving the proportion of crypts with high LGR5 expression ( $\geq 15$ LGR5 transcripts) and of crypts with LGR5 expression in upper crypt. Statistics were performed by unpaired $t$-test. Bars represent mean+SEM. Asterisks indicate significant differences ${ }^{*} p<0.05,{ }^{* *} p<0.01$, ${ }^{* * *} \mathrm{p}<0.001$. CD, Crohn's disease; IEC, intestinal epithelial cell; LYZ ${ }^{+}$, Lysozyme positive.

compared with those classified as non-inflamed (figure 2B,C). Interestingly, the number of $\mathrm{LYZ}^{+}$cells in 'upper crypt' (above +6 position) significantly increased in inflamed compared with non-inflamed tissue margins, suggesting an aberrant ISC niche architecture under inflammatory conditions (figure $2 \mathrm{~B}$, right). Reflecting the CD-like mouse model, inflamed tissue margins displayed a reduction in crypts with high $L G R 5$ expression $(\geq 15$ dots), and concomitant to $\mathrm{LYZ}^{+}$cells above the +6 position, LGR5 expression in upper crypts was induced (figure 2D,E).

\section{PANETH CELL PHENOTYPE AND LGR5-EXPRESSION PATTERN PREDICT RECURRENCE IN PATIENTS WITH CD}

As previously shown ${ }^{28}$ inflammation in resected ileal tissue at baseline was predictive for endoscopic recurrence, yet $44 \%$ of the analysed patients with $\mathrm{CD}$ with non-inflamed tissue margins still developed recurrent disease (figure 2A). Analysing regions of the intestine free of severe active or chronic inflammation has been proposed to more accurately reflect disease development due to the potential presence of early molecular and pathologic changes. ${ }^{13}$ Therefore, we tested in ileal margins classified as non-inflamed at the time of surgery if abnormal PC phenotypes and aberrant $L G R 5^{+}$expression predict endoscopic recurrence after 6-12 months. Indeed, a low proportion of highly granular PCs (figure $3 \mathrm{~A}, \mathrm{~B}$ ) and high numbers of $\mathrm{LYZ}^{+}$cells in upper crypts (figure 3C,D) correlated with the risk of recurrence, while the total number of $\mathrm{LYZ}^{+} \mathrm{PCs}$ in crypts were not different between recurrent and non-recurrent groups (online supplementary figure $2 \mathrm{~A}, \mathrm{~B})$. In line, a low proportion of crypts with high LGR5 expression (figure 3E,F) and enhanced LGR5 expression in upper crypts (figure $3 \mathrm{G}, \mathrm{H}$ ) were further predictive for endoscopic recurrence. Assigning numbers of highly granular PCs, PCs in upper crypt, highly Lgr5 expressing crypts and Lgr5 expression in upper crypts as risk factors (0-4), we demonstrated 

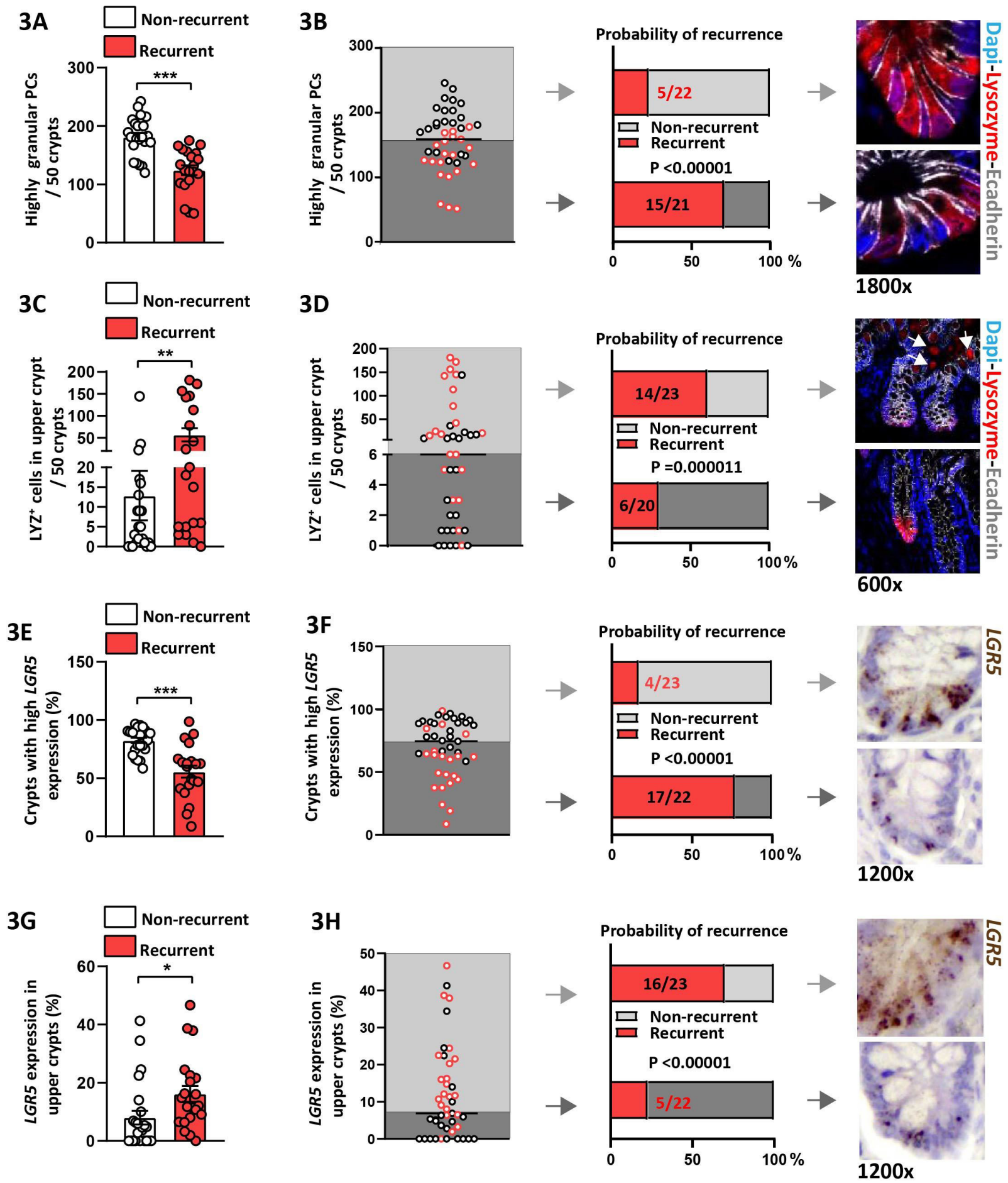

Figure 3 PC phenotype and $L G R 5$-expression predict disease recurrence in patients with non-inflamed CD Ileal tissue sections classified as noninflamed at time of surgery of patients with CD undergoing resection surgery were analysed. Tissue sections were stained for $L Y Z$ by IF and for $L G R 5$ by in situ hybridisation, respectively, and expression patterns were quantified. Numbers of highly granular LYZ ${ }^{+}$cells ( $\geq 2$ granules), numbers of LYZ ${ }^{+}$ cells in upper crypt, proportion of crypts with high LGR5 expression ( $\geq 15 L G R 5$ transcripts) and proportion of crypts with $L G R 5$ expression in upper crypt were determined. Patients with $C D$ with endoscopic recurrence (Rutgeerts score $\geq i 2$ ) 6-12 months after surgery were compared with patients with $C D$ not experiencing recurrence. $(A, C, E, G)$ Overall comparison of recurrent versus non-recurrent patients for the respective marker; $(B, D, F, H)$ From left to right: distribution of the respective marker among patients with recurrent (red circles) and non-recurrent (black circles) $C D$ with median indicated; probability of patients with $C D$ to experience recurrence if above or below the median for the respective marker; representative pictures showing sections from patients with CD above or below median. ( $A, B)$ Number of highly granular $\mathrm{LYZ}^{+}$cells ( $\geq 2$ granules), (C,D) Number of $L Y Z^{+}$ cells in upper crypt, $(B, D)$ IF costainings of Lysozyme (red) and E-cadherin (IEC borders, grey) counterstained with Dapi (nuclei, blue). (E,F) Proportion of crypts with high $L G R 5$ expression ( $\geq 15$ LGR5 transcripts), $(\mathrm{G}, \mathrm{H})$ proportion of crypts with $L G R 5$ expression in upper crypt, $(\mathrm{F}, \mathrm{H})$ LGR5 in situ hybridisation. $(A, C, E, G)$ Statistics were performed by unpaired $t$-test. Bars represent mean+SEM. Asterisks indicate significant differences * $p<0.05$, ${ }^{* *} p<0.01,{ }^{* *} p<0.001$. (B,D,F,H) Statistical analysis was performed via $\chi^{2}$ test. CD, Crohn's disease; IEC, intestinal epithelial cell; LYZ ${ }^{+}$, Lysozyme positive; PC, Paneth cell. 
a strong cumulative effect of the number of risk factors on the probability of disease recurrence (online supplementary figure 2C). Smoking, that had been identified previously as a risk factor for recurrence in this cohort, ${ }^{28}$ was associated with reduced PC granularity, a low proportion of crypts with high LGR5 expression and enhanced LGR5 expression in upper crypts (online supplementary figure $3 \mathrm{~A}-\mathrm{C}$ ). Yet, within patients with $\mathrm{CD}$ experiencing recurrence, smoking had no additional impact on the risk factors analysed (online supplementary figure 3D,E). CD-associated risk alleles of NOD2 and ATG16L1 have been reported to impact PC phenotype; ${ }^{13}$ however, we observed no impact of the single nucleotide polymorphisms ATG16L1 rs6752107 and NOD2 rs2066845 or rs2066844 on the ISC niche appearance, probably due to a high proportion of risk allele carriers (online supplementary figure $3 \mathrm{~F}-\mathrm{H})$. Contrarily, in tissue margins classified as inflamed at the time of surgery, aberrant PC phenotypes and stemness failed to better stratify disease recurrence (online supplementary figure 4). In summary, these data show a strong predictive value for alterations of the ISC niche for early endoscopic recurrence, indicating these phenotypic changes as first molecular signs of inflammatory changes, preceding macroscopic lesions.

\section{INFLAMMATION IN TNF ${ }^{\triangle A R E}$ MICE IS ASSOCIATED WITH IMPAIRED MITOCHONDRIAL FUNCTION}

Phenotypic changes of PCs have been described concomitantly to structurally impaired mitochondria, ${ }^{14}$ and mitochondrial stress signalling (MT-UPR) is apparent in IEC from patients with IBD and mouse models of intestinal inflammation. ${ }^{25}$ Accordingly, adenosine triphosphate (ATP) levels were reduced in isolated ileal crypts from $\mathrm{TNF}^{\triangle \mathrm{ARE}}$ mice, along with increased levels of the MT-UPR marker proteins Hsp60 and (dsRNAactivated protein kinase) $\mathrm{Pkr}^{25}$ under inflammatory conditions (figure 4A-D). Concomitantly, transcriptional levels of genes involved in signalling pathways related to disturbed mitochondrial function, including MT-UPR (Trb3, Atf5, Chop), antioxidative response (Hif1a) and low ATP levels (Prkaa2, AMP-kinase) were upregulated in ileal crypts (figure 4E), while expression of Grp78, a surrogate marker of ER stress remained unaltered (figure 4F). In line, transmission electron microscopy showed markedly reduced numbers of PCs with typical morphological features at the ileal crypt bases of inflamed TNF ${ }^{\Delta A R E}$ mice. The few remaining Paneth-like cells often exhibited secretory granules with broadened halos, intracytoplasmic vacuolations, dilation of the rough ER, as well as degenerative mitochondrial alterations, including mitochondrial swelling with dissolution and disruption of cristae, loss of matrix density and occasionally formation of intramitochondrial electron-dense inclusions (figure 4G). Autophagy contributes to the mobilisation of cellular energy stores and alterations in autophagy-related proteins have been linked to the disruption of the PC granule exocytosis pathway and degenerating mitochondria. ${ }^{14}$ Thus staining for the autophagy marker LC3, an increase in LC3 expression in crypt bases of inflamed TNF ${ }^{\triangle A R E}$ mice was observed accompanying metabolic and morphologic alterations (online supplementary figure $5 \mathrm{~A}, \mathrm{~B})$.

\section{MITOCHONDRIAL IMPAIRMENT IN ISC CAUSES TRANSITION TOWARDS DYSFUNCTIONAL PANETH CELLS}

PC and $\operatorname{Lgr5}^{+}$ISC alterations concurrent to mitochondrial impairment were present under inflammatory conditions in $\mathrm{TNF}^{\triangle \mathrm{ARE}}$ mice. Hence, we characterised the role of mitochondrial function on ISC niche appearance using a mouse model in which Hsp60 can be specifically deleted in $\operatorname{Lgr} 5^{+}$ISC $\left(H s p 60^{\text {flox/ }}\right.$ flox $\times \operatorname{Lgr} 5$-eGFP-IRES-CreER $\left.{ }^{\mathrm{T} 2-\mathrm{Tg}}\right)$ via administration of tamoxifen $\left(\mathrm{Hsp} 60^{\Delta / \Delta \mathrm{ISC}}\right) \cdot{ }^{26} \mathrm{Hsp} 60$ represents a target gene of MT-UPR signalling and constitutes the main chaperone of the mitochondrial matrix. ${ }^{30}$ Thus, loss of Hsp60 leads to disturbed proteostasis in mitochondria and subsequent activation of MT-UPR signalling. ${ }^{26}$ Consequently, Hsp60 deficiency results in mitochondrial dysfunction, including reduced mitochondrial respiration and a drop in cellular ATP content. ${ }^{26}$ Confirming MT-UPR activation on Hsp60 loss, transcription of the cochaperone and surrogate marker of MT-UPR, Hsp10 increased early after induction of Hsp60 deletion in parallel with induction of Trb3, Chop, Hif1a and Prkaa2 (figure 5A). Following Lgr5 and Lyz expression in the ileum of $\mathrm{Hsp} 60^{\Delta / \Delta I S C}$ mice for up to 6 days after the end of tamoxifen treatment, transient changes of the ISC niche were characterised. At day 2, the proportion of highly Lgr5 expressing crypts dropped (figure 5B,C). Parallel efforts to characterise PC phenotypes demonstrated that $\mathrm{Lgr}^{-} \mathrm{Lyz}^{+}$cell numbers decreased, while numbers of $\mathrm{Lgr}^{+} \mathrm{Lyz}^{+}$double positive cells increased, starting at day 0 after end of tamoxifen treatment (figure 5B-D). Along with diminished expression of markers for ISCs and PCs, PC granularity and expression of PC-derived AMPs were reduced in response to Hsp60 loss-induced mitochondrial dysfunction in ISCs (figure 5E-G). Reflecting inflamed $\mathrm{TNF}^{\mathrm{ARE}}$ mice, LC3 staining in crypt bases of Hsp60 $0^{\Delta / \Delta \mathrm{ISC}}$ mice depict induction of autophagy (online supplementary figure $5 \mathrm{C}$ ) along with metabolic and morphological alterations of the ISC niche. To exclude the possibility of mitochondrial dysfunctionmediated cell death of $\mathrm{Lgr}^{+}$ISC and $\mathrm{Lyz}^{+}$cells, tissue sections from day 2, when loss of Lgr5 expression was most pronounced, were costained for TUNEL and eGFP or Lyz, respectively. Furthermore, tissue sections were stained for the apoptosis marker cleaved caspase (CC) 3, confirming absence of enhanced apoptosis at day 2 after the end of tamoxifen treatment, but indicating increased apoptosis in crypt basis at days 4 and 6 (online supplementary figure 6). Hsp60 deficiency in $\operatorname{Lgr}^{+}$ISCs was associated with a complete loss of proliferation as indicated by Ki67 staining (online supplementary figure 7A). However, expression of Olfm 4 and Hopx, a marker of +4 reserve stem cells potentially contributing to $\operatorname{Lgr} 5^{+}$ISC replenishment, ${ }^{31}$ was retained and transiently enhanced in crypts (online supplementary figure $7 \mathrm{~B}, \mathrm{C}$ ). In line, cells above the crypt basis remained Hsp60 and Ki67 positive throughout the observed time points (online supplementary figure 7A). Crypt-based cells regained Hsp60 and Ki67 expression from day 4 on, indicating apoptotic cell death and extrusion of Hsp60 deficient cells. Lgr $5^{+}$ ISC can differentiate into PCs, and vice versa, dedifferentiation of PCs and acquisition of stem-like features has been described following loss of CBC ISCs. ${ }^{910}$ To determine if dysfunctional PCs arise from mitochondrial function-impaired ISCs, we stained for Lyz and Hsp60. Hsp60 negative cells directly originated from $\mathrm{Lgr5}^{+}$ISCs that had suffered from mitochondrial dysfunction. The ratio of $\mathrm{Lyz}^{+}$cells in which Hsp60 could not be detected was increased at day 0 and day 2 (figure 5H,I), indicating $\operatorname{Lgr} 5^{+}$, Hsp60- ISCs to acquire Lyz expression via mechanisms initiated by mitochondrial dysfunction. Furthermore, reduced PC granularity and diffuse Lyz staining was associated with lack of Hsp60 in PCs (figure 5J). In summary, these data suggest that mitochondrial impairment, including reduced mitochondrial respiration, initiates a transition of $\mathrm{Lgr} 5^{+}$ISCs towards a PC-like phenotype. However, the inability of these $\mathrm{Lgr}^{+} \mathrm{Lyz}^{+}$double positive cells to adjust their mitochondrial function to the new cell-phenotypic demand (due to lack of Hsp60), seems to hamper differentiation into functionally mature PCs (figure $5 \mathrm{~K}$ ). Interestingly, costaining 


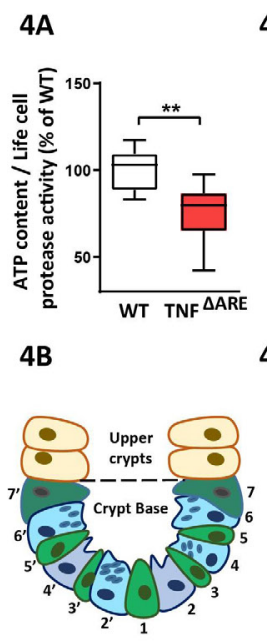

4E

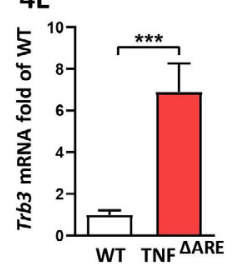

$4 \mathrm{G}$

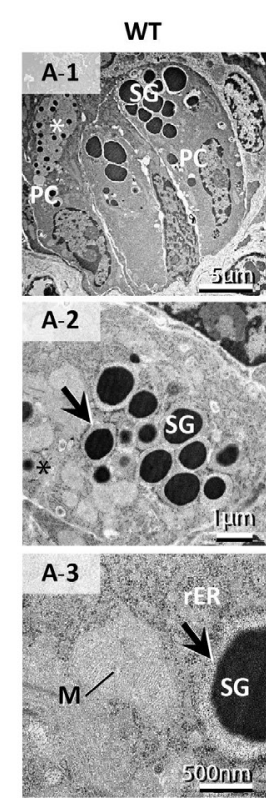

$4 C$
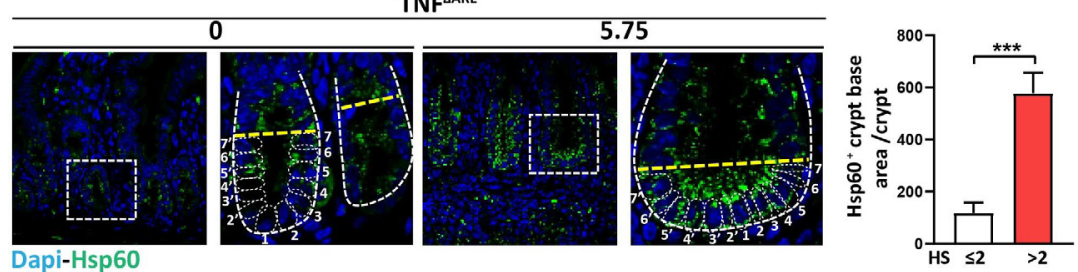

TNFAARE
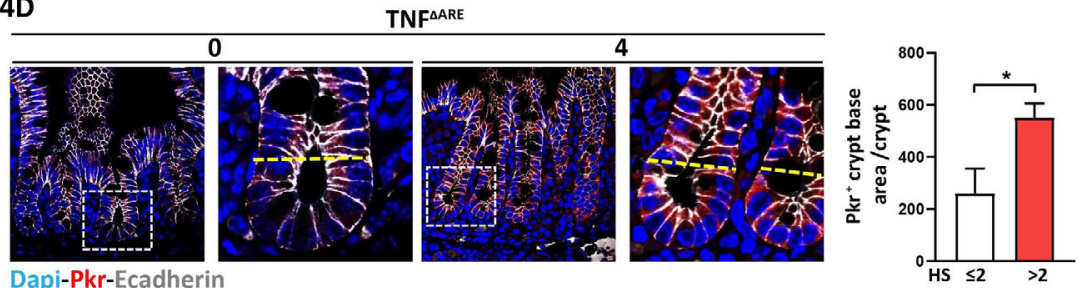

4F
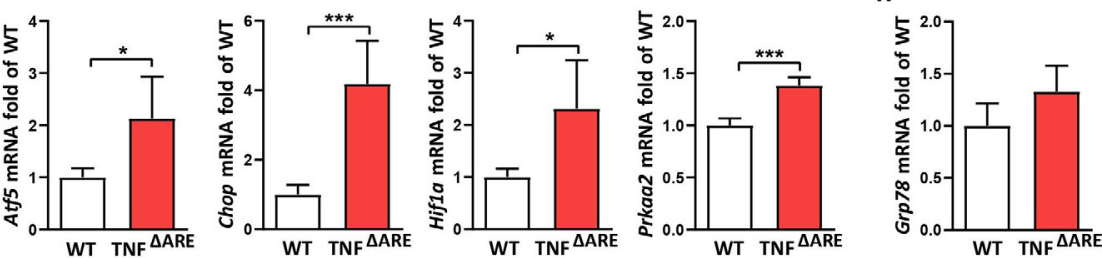

Figure 4 Inflammation in TNF ${ }^{\triangle A R E}$ mice is associated with mitochondrial dysfunction in ileal crypts Isolated ileal crypts and tissue sections from $T_{N N F}{ }^{\triangle A R E}$ mice and WT littermates were analysed. (A) ATP content of primary isolated crypts relative to life cell protease activity measured by a fluorescence assay ( $n=10 / 7)$. (B) Overview of crypt structure and illustration of the area used for protein quantification. (C) IF images of Hsp60 (green) counterstained with Dapi (nuclei, blue), including magnifications. Numbers above the pictures indicate the HS of the respective tissue section. Right: corresponding quantification. (D) Costaining of Pkr (green) and E-cadherin (IEC borders, grey) counterstained with Dapi (nuclei, blue), including magnifications. Numbers above the pictures indicate the HS of the respective tissue section. Right: corresponding quantification. (E) qRT-PCR analysis of primary ileal crypts for genes involved in mitochondrial MT-UPR, mitochondrial signalling and (F) ER stress ( $n=6)$. Statistics were performed by unpaired $t$-test. Bars represent mean+SEM. Asterisks indicate significant differences ${ }^{*} p<0.05,{ }^{* *} p<0.01,{ }^{* *} p<0.001$. (G) Transmission electron microscopy of ileal crypt bases. Panel A: WT; A1-2: PCs display abundant, apical, electron-dense SGs with narrow halos (arrow). Asterisks mark secretory granules with small electron dense cores and wide rims of flocculent material of low electron-density. $A-3$. Unaltered appearance of rER and mitochondria (M) in a WT-PLC. B: non-inflamed TNF ${ }^{\triangle A R E}$ mice; $B-1-6$ : PC ultrastructure essentially resembles WT mice. N: nucleus. $C$ : inflamed TNF $^{\triangle A R E}$ mice. C-1-2: Few remaining cells with PLC-typical location and morphology often show vacuolation (V) and broadened halos of secretory granules. IC: infiltrating inflammatory cell C-4-6: Mitochondrial lesions in PLC include intramitochondrial inclusions (C-4, C-5), mitochondrial swelling with dissolution and disruption of cristae, and loss of matrix density and also distension of the rER (C-6). ARE, AU-rich (adenosin-uracil) elements; $C D$, Crohn's disease; HS, histopathological score; IEC, intestinal epithelial cell; PC, Paneth cell; rER, rough endoplasmic reticulum; SG, secretory granule; TNF, tumour necrosis factor; WT, wild type. 

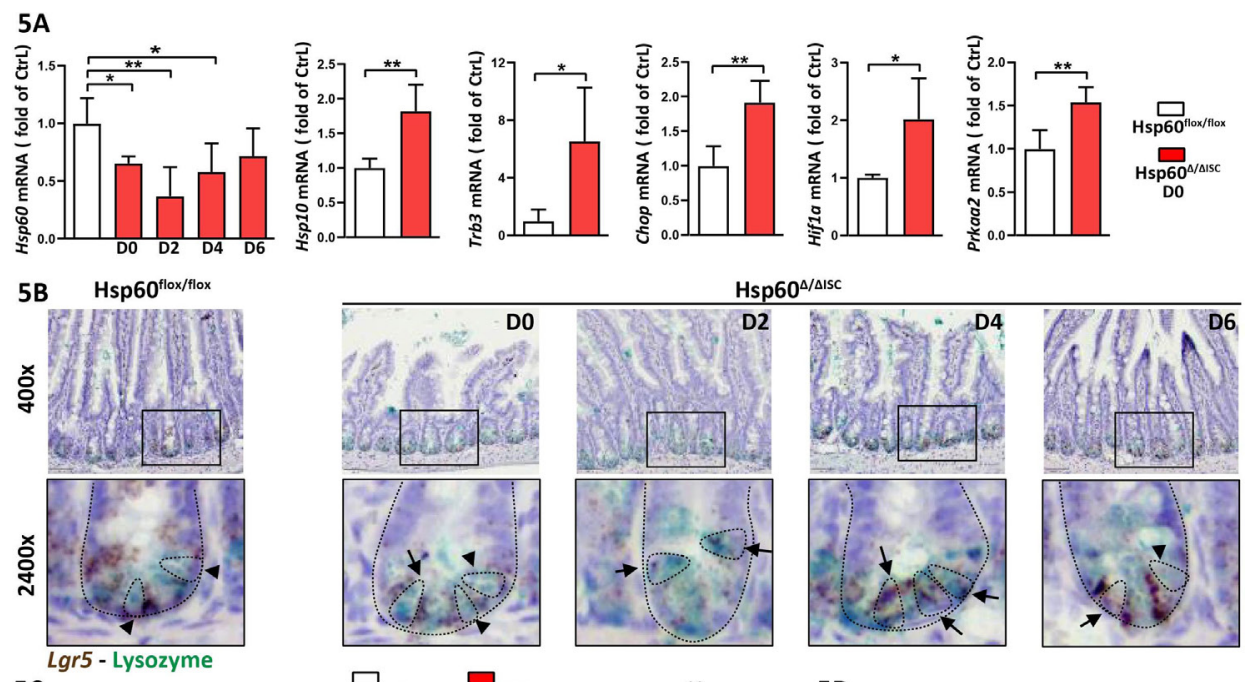

$5 \mathrm{C}$
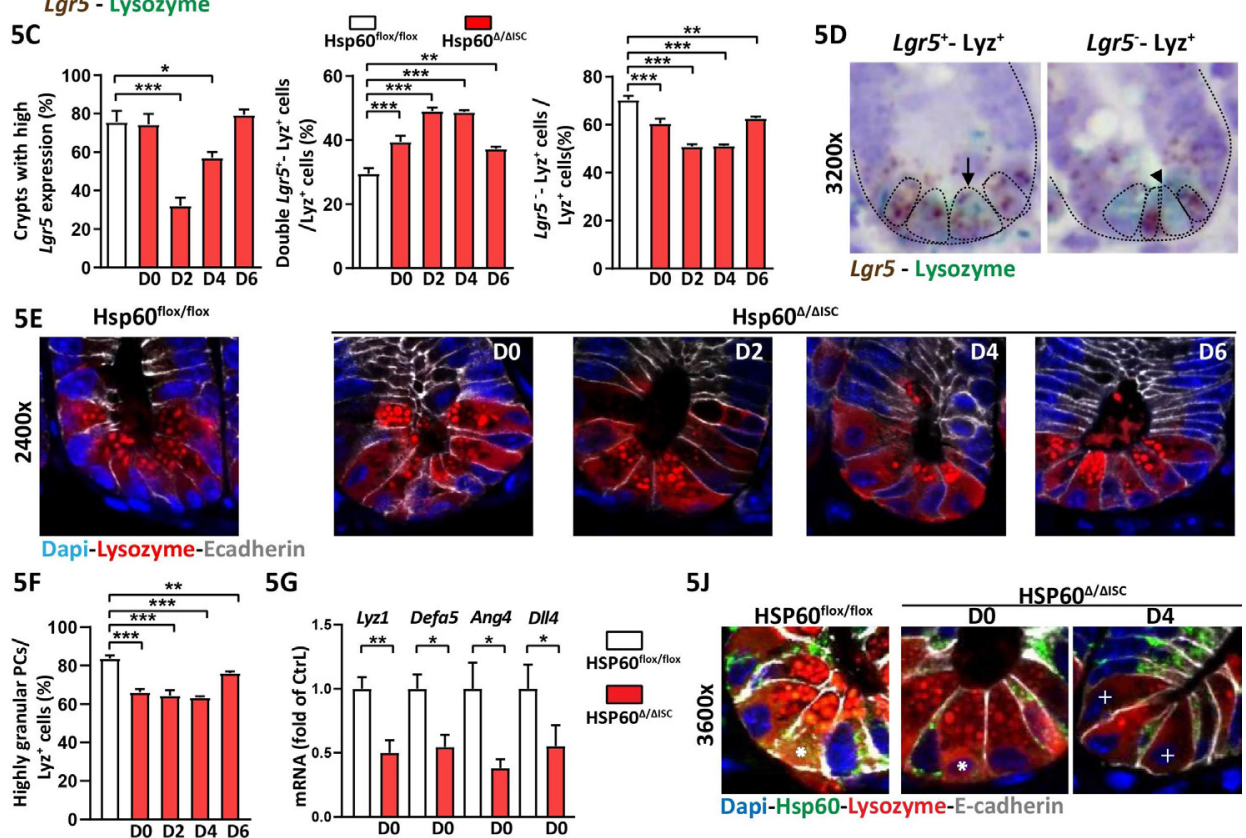

$5 G$
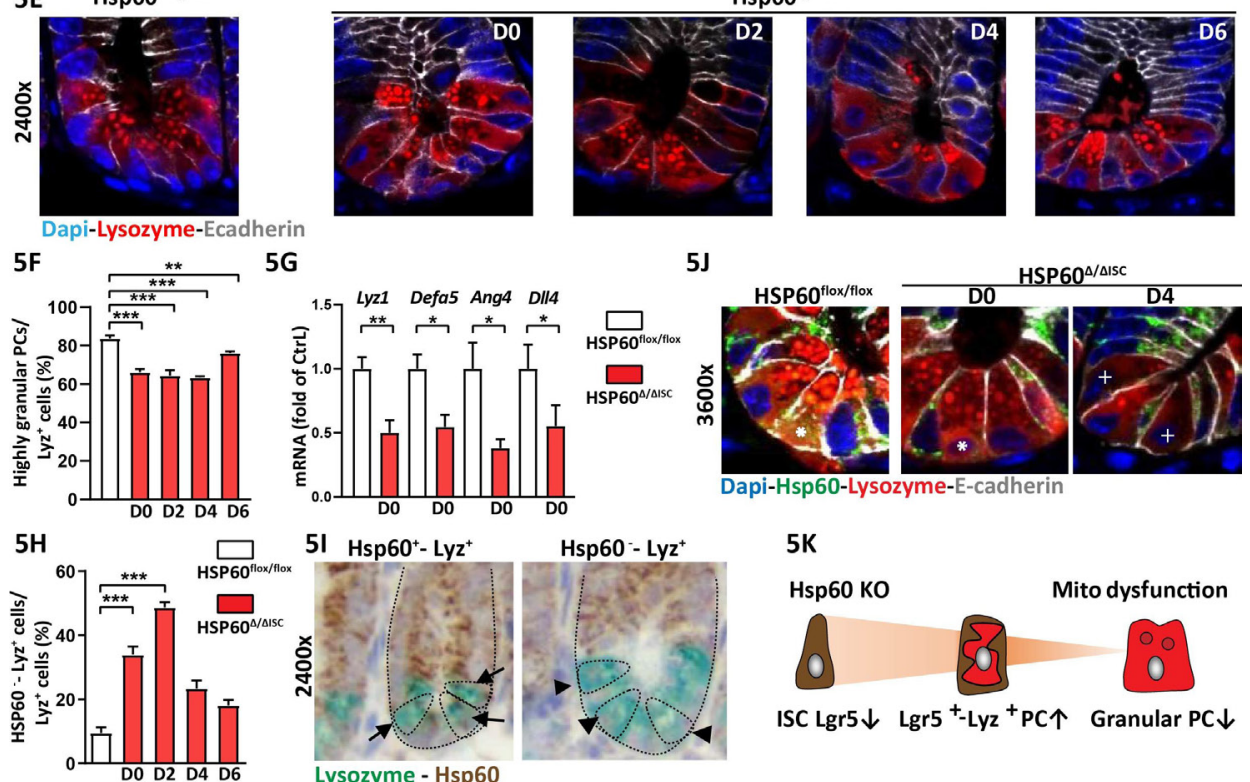

Figure 5 Mitochondrial impairment in ISC causes transition towards dysfunctional Paneth cells Ileal tissue sections and IEC isolates of Hsp60 flox/ flox mice and $\mathrm{Hsp60}{ }^{\Delta / \Delta \mathrm{ISC}}$ mice were analysed at different time points after end of tamoxifen treatment. (A) qRT-PCR analysis of IECs for Hsp60 and genes involved in mitochondrial MT-UPR and mitochondrial signalling at day 0. (B) Lgr5 in situ hybridisation (brown) and lysozyme (Lyz, turquoise) immunehistochemistry (IHC) costaining, lower panel: magnifications, dotted lines indicate crypt and cell borders, arrow-heads indicate Lyz ${ }^{+}$cells, arrows indicate Lgr5-Lyz double-positive cells. (C) Quantification of the proportion of crypts with high Lgr5 expression ( $\geq 10$ Lgr5 transcripts), the proportion of $\operatorname{Lgr} 5$ - Lyz double-positive cells, and the proportion of $\operatorname{Lgr} 5$ negative, Lyz single-positive cells over time. (D) Representative pictures of Lgr5-Lyz double-positive cells, and Lgr5 negative, Lyz single-positive cells; dotted lines indicate crypt and cell borders, arrows indicate Lgr5-Lyz double-positive cells, arrow-heads indicate Lgr5 negative, Lyz single-positive cells. (E) IF costaining of Lyz (red) and E-cadherin (IEC borders, grey) counterstained with Dapi (nuclei, blue) showing granular and non-granular staining pattern. (F) Quantification of the proportion of highly granular Lyz $z^{+}$cells ( $\geq 2$ granules). (G) qRT-PCR analysis of IECs from day 0 for PC-derived AMP and DII4. (H) Quantification of the proportion of Hsp60 negative, Lyz single positive cells. (I) Representative pictures of IHC costaining for Hsp60 (brown) and Lyz (turquoise) used for quantification; dotted lines indicate crypt and cell borders, arrow indicates Hsp60 — Lyz double positive cells, arrow-heads indicate Hsp60 negative, Lyz single-positive cells. (J) IF costaining of Hsp60 (green), Lyz (red), and E-cadherin (IEC borders, grey) counterstained with Dapi (nuclei, blue), including magnifications. Asterisks indicate Hsp60-Lyz double positive, highly granular Paneth cells, crosses indicate Hsp60 negative, Lyz single-positive cells, depicting a diffuse Lyz staining. (K) Schematic representation of the main findings in this figure. Statistical analyses were performed by one-way ANOVA followed by Tukey test or unpaired $t$-test. Bars represent mean+SEM. Asterisks indicate significant differences ${ }^{*} p<0.05,{ }^{* *} p<0.01,{ }^{* * *} p<0.001$. AMP, antimicrobial peptides; ANOVA, analysis of variance; IEC, intestinal epithelial cell; MT-UPR, mitochondrial unfolded protein response; 
of Hsp60 with Mucin 2 (Muc2), Chromogranin A (ChgA) or doublecortin-like kinase 1 ( $\mathrm{dclk} 1)$ as markers for the secretory IEC types (goblet cells, enteroendocrine cells and tuft cells, respectively) indicated that ISC suffering from mitochondrial dysfunction only acquired Lyz expression, since Muc2, ChgA and dclk1 expressing cells remained Hsp60 positive (online supplementary figure $8 \mathrm{~A}-\mathrm{C}$ ). At day 6 after tamoxifen treatment, ISCs and PCs regained normal phenotypes comparable to $H s p 60^{\text {flox/flox }}$ mice (figure 5B,C,E,F,H). At this time point, Hsp60 mRNA expression in IEC returned to control levels (figure $5 \mathrm{~A}$ ). The complete regain of $\mathrm{Hsp} 60^{+}, \mathrm{Lgr} 5^{+}$ISCs and a normal ISC niche phenotype indicates replenishment of $\mathrm{Lgr}^{+}$ISCs from reserve ISC populations as described before. ${ }^{932}$

\section{CRYPTS FROM INFLAMED TNF ${ }^{\triangle A R E}$ MICE FAIL TO GROW INTO ORGANOIDS}

Further investigating the role of mitochondrial function on ISC niche-regulation under inflammatory conditions, primary crypts of non-inflamed and inflamed $\mathrm{TNF}^{\mathrm{ARE}}$ mice were isolated and seeded in medium without Wnt factors. ${ }^{33}$ Addition of Wnt factors to the medium is not essential for small intestinal (SI) organoid culture and has been linked to the presence of PCs providing these signals naturally. ${ }^{34}$ Ileal crypts from inflamed mice displayed reduced Lgr5 and Lyz expression, confirming figure 1 (figure 6A,B). Consistently, ileal crypts from inflamed, but not non-inflamed $\mathrm{TNF}^{\triangle \mathrm{ARE}}$ mice almost completely failed to grow into organoids. Remaining organoids showed a severe defect in de-novo crypt formation (budding) (figure 6C). In noninflamed TNF ${ }^{\mathrm{ARE}}$ mice and WT littermates, seeding efficiency and budding were comparable (online supplementary figure 9A), confirming inflammation and not genotype as responsible for impaired growth. Of note, crypts derived from the jejunum, directly adjacent to the inflamed ileum but showing no tissue pathology in $\mathrm{TNF}^{\triangle \mathrm{ARE}}$ mice, also displayed a reduced ability to form organoids (figure 6D). Applying an intestinal organoid culture medium supplemented with Wnt factors directly after crypt isolation did not rescue growth (online supplementary figure 9B). Hence, it is likely that disturbances of both $\mathrm{Lgr}^{+}$ ISCs and PCs contribute to the inflammation-induced growth defect.

\section{MITOCHONDRIAL RESPIRATION IS REQUIRED TO MAINTAIN STEMNESS AND PC FUNCTIONALITY}

The metabolism of cells constituting the ISC niche is coordinated to support each other's demand and particularly mitochondrial oxidative phosphorylation (OXPHOS) is fine tuned to meet the requirements of different IEC subtypes. ${ }^{8}{ }^{24}$ Since crypts derived from an inflammatory environment and $\mathrm{Hsp} 60^{\Delta / \Delta I S C}$ organoids both display mitochondrial dysfunction, ISC niche alterations and a growth defect (figures 4 and 6A-D, online supplementary figure 9C,D), we determined mRNA expression levels of key factors involved in OXPHOS and glycolysis in primary crypt derived from non-inflamed and inflamed $\mathrm{TNF}^{\triangle \mathrm{ARE}}$ mice.

Pdha, belonging to the pyruvate dehydrogenase complex (PDC), Yy1, a transcriptions factor regulating mitochondrial complex I genes and cytochrome coxidase (Cox) IV were consistently reduced (figure 6E). PDC acts as gatekeeper of metabolism by linking cytoplasmic glycolysis to the TCA cycle and OXPHOS, Hence, oligomycin, blocking OXPHOS and dichloroacetate (DCA), targeting PDC to shift ATP generation from glycolysis to OXPHOS ${ }^{35}$ (figure 6F), were used to characterise the role of metabolism on ISC niche function. Murine ileal WT organoids and human SI organoids were treated with sublethal doses of oligomycin (online supplementary figure 9E) or DCA. Organoids treated with oligomycin depict lower numbers of $\mathrm{Lyz}^{+}$cells per crypt and reduced PC granularity (figure 6G). Concomitantly, transcriptional levels of Lgr5, Lyz and Ang4 were reduced, while Chop, Hif1a and Prkaa were upregulated (figure 6G). Noteworthy, these results were mirrored in human intestinal organoids (figure $6 \mathrm{H}$ ). In contrast, DCA had only minimal effects on these readouts (online supplementary figure 10).

\section{REINFORCING MITOCHONDRIAL RESPIRATION RESTORES INFLAMMATION-IMPRINTED DYSFUNCTION OF THE ISC NICHE}

Finally, we tested if the inflammation-associated growth defect in $\mathrm{TNF}^{\triangle A R E}$ mice-derived crypts could be reversed by DCA. Indeed, addition of DCA to the organoid culture medium directly after seeding rescued the ability of inflamed $\mathrm{TNF}^{\mathrm{ARE}}$ mice-derived ileal crypts to grow into organoids and form de-novo crypts (figure 6J). Strikingly, DCA withdrawal subsequent to passaging at day 8 of culture conferred no negative effects to organoid growth compared with continued DCA treatment (figure 6K,L). Comparing DCA-exposed organoids derived from inflamed $\mathrm{TNF}^{\triangle A R E}$ mice and WT mice, transcriptional level of ISC and $\mathrm{PC}$-associated genes largely converged (figure 6M). These data demonstrate that activation of OXPOHOS via inhibition of glycolysis is sufficient to restore inflammation-imprinted metabolic dysfunction of the ISC niche.

The persisting regain of stemness after withdrawal of DCA underlines the reversible nature of ISC alterations under inflammatory conditions, making ISC niche metabolism an attractive target for therapeutic interventions (figure 7).

\section{Discussion}

IBDs, including CD and ulcerative colitis, constitute a global health problem. ${ }^{36} \mathrm{~A}$ major challenge in the treatment of $\mathrm{CD}$ is the heterogeneity of the disease, with only subsets of patients responding to therapies like administration of anti-TNF antibodies. $^{37}$ The great number of susceptibility genes identified along with a range of different environmental triggers, might account for these difficulties, implicating diverse mechanisms contributing to the variable phenotypes of CD. Several CD-relevant pathways converge on the level of PCs, like ER stress, autophagy and bacterial recognition and consistently, the presence of NOD2 and ATG16L1 disease-associated alleles as well as bacterial infection have been demonstrated to affect PC phenotype. ${ }^{12141538}$ Hence, aberrant PC phenotypes have been proposed as a biomarker to stratify patients with $\mathrm{CD}$ according to similar disease mechanisms in order to yield better treatment outcomes. ${ }^{13}$ The data presented here implicate that determining ISC niche appearance improves risk stratification of patients with $\mathrm{CD}$, and that targeting the underlying mitochondrial dysfunction evolves as a novel strategy for therapy.

Reduced PC functionality has been extensively described for $\mathrm{CD}$ in the context of AMP production and alterations of the intestinal microbiota. ${ }^{351527}$ However, PCs serve as multifunctional guardians of the crypt, also providing essential signals for the ISC niche. ${ }^{27}$ PCs evolve directly above the crypt base, and $\mathrm{Lgr}^{+}$ISCs compete for available surface of their own direct progeny, ${ }^{34}$ implicating ISC changes concomitant to PC abnormalities. Consequently, we found diminished Lgr 5 expression in patients with $C D$ and in inflamed $T_{N F}{ }^{\triangle A R E}$ mice, reflecting the inflammation-associated gradual loss of PC function. Furthermore, in patients with CD, LYZ and LGR5 showed a distorted 
$6 \mathrm{~A}$

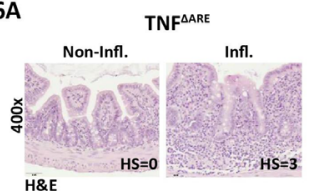

H\&E

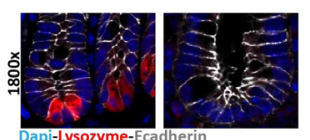

6B

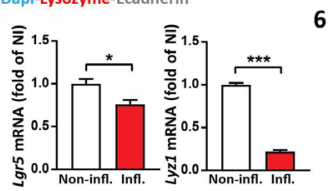

6C

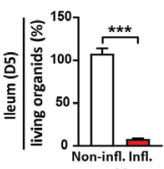

6D

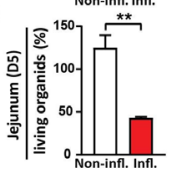

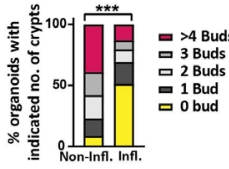

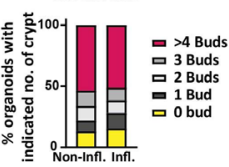

$6 \mathrm{E} \overline{\bar{z}}$
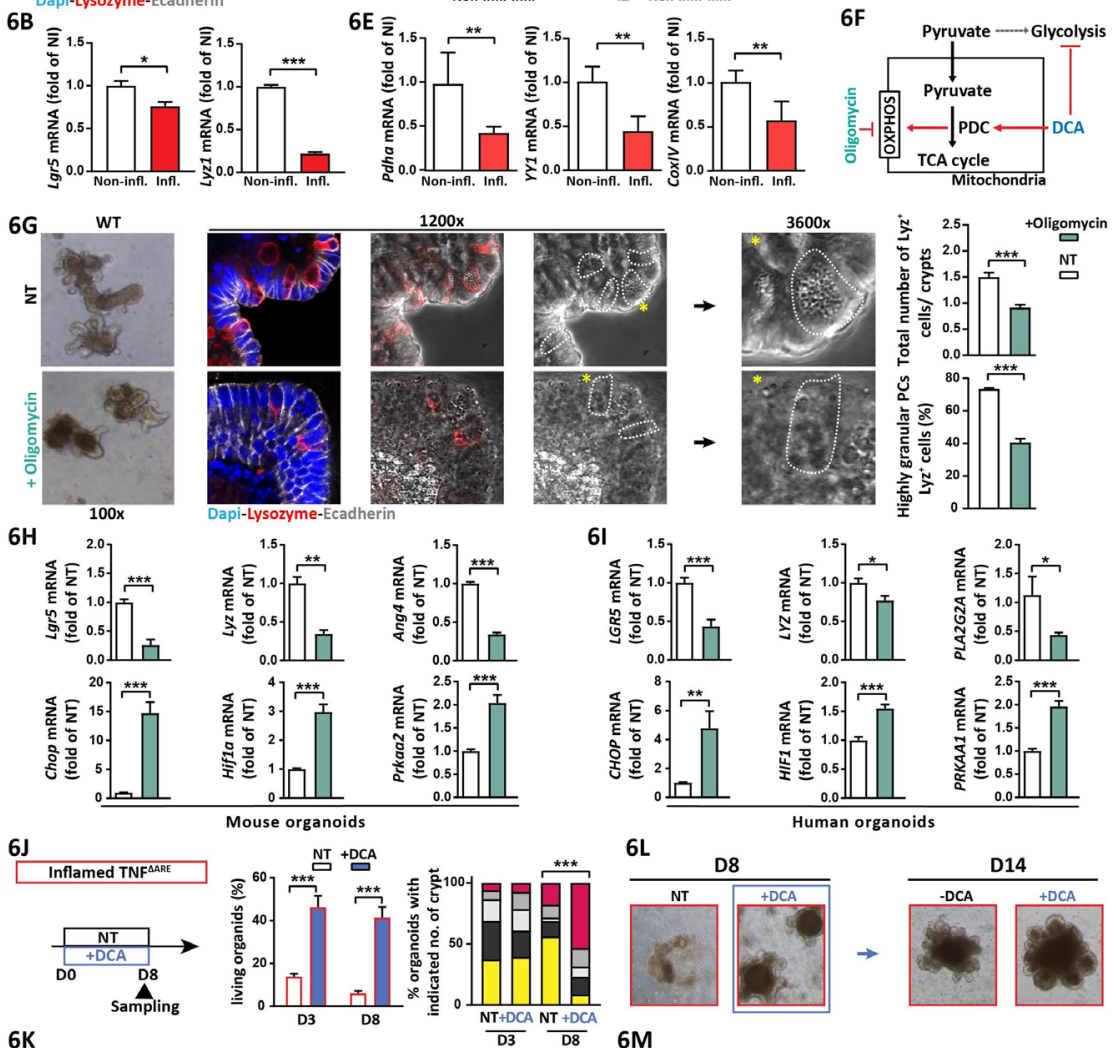

6L

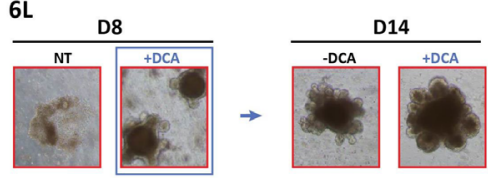

$6 \mathrm{~K}$
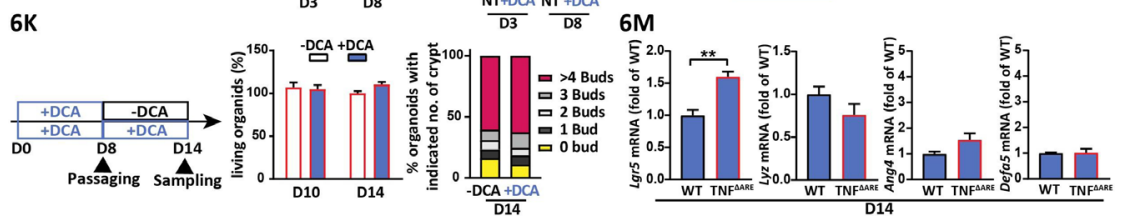

Figure 6 Mitochondrial respiration determines stemness and PC functionality under inflammatory conditions (A-E) lleal tissues and crypts derived from non-inflamed and inflamed TNF ${ }^{\triangle A R E}$ mice were analysed. (A) Representative pictures of ileal sections; top: H\&E staining with histopathological score indicated; bottom: IF costaining of Lyz (red) and E-cadherin (grey) counterstained with Dapi (nuclei, blue) showing absence of PCs in inflamed tissue. (B) qRT-PCR analysis of primary ileal crypts for $L g r 5$ and $L y z(n=6)$. (C,D) Characterisation of intestinal organoids derived from ileal $(C)$ and jejunal (D) crypts at day 5 of ex vivo culture. Left: Proportion of living organoids; middle: quantification of de novo crypt formation (budding); right: representative bright field pictures. (E) qRT-PCR analysis of primary ileal crypts for metabolism-determining genes ( $n=6$ ). (F) Schematic representation of oligomycin and DCA targets. $(\mathrm{G}, \mathrm{H})$ Ileal organoids derived from WT mice were treated with oligomycin for 24 hours. (G) Left: bright field and IF costaining of Lyz (red) and E-cadherin (IEC borders, grey) counterstained with Dapi (nuclei, blue). Dotted lines indicate cell borders of Lyz ${ }^{+}$cells; asterisks indicate Lyz ${ }^{+}$cells magnified in the pictures on the right side. Right: quantification of Lyz ${ }^{+}$cell numbers per crypt (upper graph) and the proportion of highly granular ( $\geq 2$ granules) Lyz ${ }^{+}$cells (lower graph). (H) qRT-PCR analysis of intestinal organoids for $L g r 5$ and Paneth cell functionassociated genes (upper panel) and for genes associated with mitochondrial signalling (lower panel, $n=6)$. (I) Same analysis as in (H) for human organoids derived from the small intestine and treated with oligomycin for 24 hours $(n=6)$. (J-L) lleal organoids derived from inflamed TNF ${ }^{\triangle A R E}$ mice were analysed $(n=6)$. (J) From left to right: experimental scheme (D0=isolation of primary crypts and start of culture), non-treated and DCA-treated organoids were compared; proportion of living organoids at day 3 and day 8 of culture; quantification of de novo crypt formation at day 3 and day 8 . (K) From left to right: experimental scheme, DCA-treated organoid cultures were passaged at day 8, and subsequently cultured in control (-DCA) and DCA-containing medium, respectively, until day 14; proportion of living organoids at day 10 and day 14 of culture; quantification of de novo crypt formation at day 14. (L) Representative bright field pictures of organoids at day 8 (before passaging) and at day 14 of culture. (M) WT and inflamed $\mathrm{TNF}^{\triangle \mathrm{ARE}}$ mice-derived ileal organoids were treated with DCA for 14 days. qRT-PCR analysis for $\operatorname{Lgr} 5$ and Paneth cell function-associated genes.

Statistics were performed by unpaired $t$-test when comparing two groups and by one-way ANOVA followed by Tukey test for three-group comparisons, respectively. Statistics for budding analyses were performed by Kruskal-Wallis test on ranks. Bars represent mean+SEM. Asterisks indicate significant differences ${ }^{*} p<0.05,{ }^{* *} p<0.01,{ }^{* * *} p<0.001$. ANOVA, analysis of variance; ARE,AU-rich (adenosin-uracil) elements; DCA, dichloroacetate; Lyz ${ }^{+}$, Lyz positive; OXPHOS, oxidative phosphorylation; WT, wild type. 


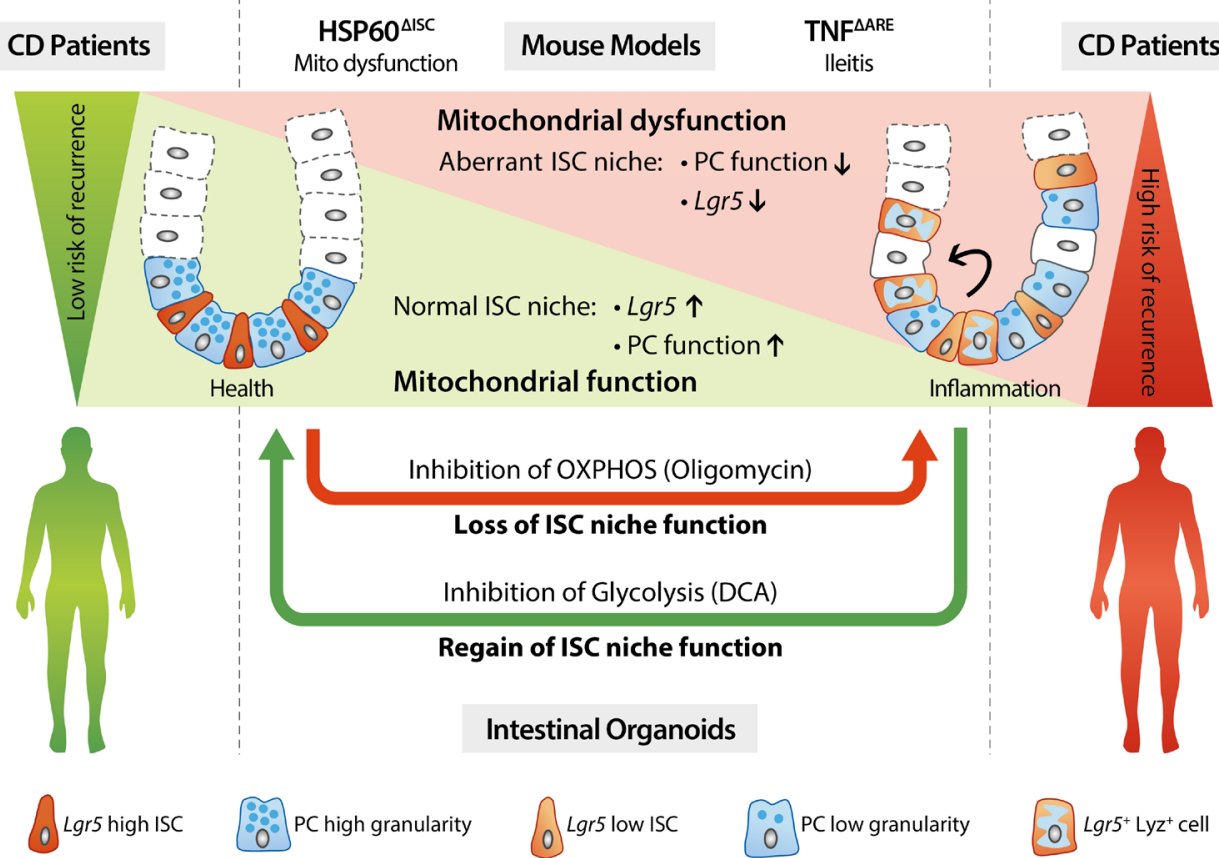

Figure 7 Mitochondrial impairment drives ISC transition towards dysfunctional PCs predicting Crohn's disease recurrence Schematic representation of the main findings of this work. Targeted disruption of mitochondrial function in ISCs leads to transition of ISCs into dysfunctional PCs. Under inflammatory conditions, mitochondrial impairment in the ISC niche results in ISC exhaustion and generation of dysfunctional PCS characterised by loss of Lyz positive granules, concomitant to aberrant Lgr5 and Lyz expression in upper crypts. These alterations precede tissue pathology and serve as predictive markers for early endoscopic recurrence in CD. Ex vivo, the inhibition of mitochondrial respiration (OXPHOS) in intestinal organoids reflects the impact of an inflammatory environment on the ISC niche, whereas reinforcement of OXPHOS by inhibition of glycolysis is able to override inflammation-imprinted changes of the ISC niche. ARE,AU-rich (adenosin-uracil) elements; CD, Crohn's disease; ISC, intestinal stem cell; Lyz, Lysozyme; OXPHOS, oxidative phosphorylation; PC, Paneth cell; TNF, tumour necrosis factor.

expression pattern with PCs and ISCs abundantly present in upper crypts. So far, $\mathrm{Lgr}^{+}$ISC loss has been described in the primary response to DSS-induced inflammation and on highdose $\gamma$-irradiation. ${ }^{9}{ }^{39}$ The temporary depletion and rapid restoration of these cells following acute injury seems to underlie the regenerative response of crypts. Under these conditions, PCs contribute to the repair and regeneration of damaged intestinal tissue by acquiring stem like features. ${ }^{9-11}$ However, irradiation and DSS-induced inflammation cause acute mucosal injury and do not reflect the chronic inflammatory conditions of IBD pathology. Despite reduced Lgr5 expression, crypts derived from DSS-treated mice show an enhanced capacity to form organoids, ${ }^{9}$ in contrast to crypts from inflamed $\mathrm{TNF}^{\mathrm{ARE}}$ mice that fail to give rise to organoids. Hence, the phenotypic changes of the ISC niche under chronic inflammation are likely not associated with healing processes but rather indicate stemness exhaustion as a feature of pathology. Moreover, ISC niche abnormalities were detected before onset of severe tissue pathology in TNF $\mathrm{TNRE}^{\triangle \mathrm{ARE}}$ mice and in non-affected tissue margins of patients with CD, suggesting that these changes most likely represent early, molecular marker of inflammatory changes.

Additionally, we provide experimental evidence for a direct role of mitochondria in the development of dysfunctional PCs using an ISC-specific mouse model of mitochondrial dysfunction and ex vivo inhibition of OXPHOS in organoid cultures. Experimental disturbance of mitochondrial function reduced stemness and PC functionality, demonstrating dysfunctional PCs as direct descendants of ISCs suffering from mitochondrial impairment. Thus, while mitochondrial dysfunction in ISC seems to disturb the balance between self-renewal and differentiation, the inability to fine-tune mitochondrial function subsequently seems to interfere with differentiation processes, impeding normal PC maturation.

This is in line with the pivotal role of mitochondria in ISC fate through regulation of the metabolic switch during differentiation. ${ }^{24}$ In $\mathrm{Lgr}^{+}$ISCs isolated from the mouse small intestine, OXPHOS highly contributes to cellular bioenergetics, and mitochondrial activity further increases with differentiation and de-novo crypt formation in intestinal organoids, ${ }^{8}$ indicating why ISC and ISC differentiation processes might be particularly vulnerable to mitochondrial impairment. OXPHOS inhibition and the subsequent decrease in ISC and PC marker genes was associated with transcriptional activation of Chop, hypoxiainducible factor $1-\alpha$ and AMP kinase in murine and human intestinal organoids. All of these genes are involved in metabolic regulation and link mitochondrial homeostasis to CD-relevant pathomechanisms including MT-UPR, ROS signalling and energy sensing. ${ }^{24} 40-42$

Most remarkable, ex vivo culture experiments using intestinal crypts derived from inflamed $\mathrm{TNF}^{\triangle \mathrm{ARE}}$ mice suggest (I) that the inflammatory environment imprints the ISC niche towards reduced stemness and PC function, (II) that these changes persist under normal culture conditions and cannot be overcome by addition of Wnt factors, (III) that a targeted metabolic intervention using DCA treatment rescues stemness and reverses the inflammatory imprinting, allowing organoids to propagate under normal culture conditions. DCA treatment results in diminished glycolysis and improved mitochondrial respiration, an ability used in the therapy of several different solid tumours to reverse the Warburg effect in cancer cells. ${ }^{35}$ Identification of target metabolites conferring to ISC niche homeostasis could be a promising target of future research. 
The cause of mitochondrial disturbances present in patients with CD and mouse models of intestinal inflammation is currently unknown. In general, enterocytes of patients with IBD have been reported to display swollen mitochondria with irregular cristae indicative of impaired function, ${ }^{43}$ and inflammation is associated with hypoxia. Infiltrating immune cells, invading pathogens and the increased energy demand of resident cells limit the available oxygen, ${ }^{44}$ and together with reduced blood supply, ${ }^{45}$ these changes contribute to the hypoxic conditions under chronic inflammation. Furthermore, polymorphisms in genes impacting mitochondrial functions, mitochondrial carrier protein uncoupling protein (UCP) 2 and SLC22A5, encoding the carnitine transporter OCTN2, have been described as risk factors in IBD. ${ }^{46}{ }^{47} \mathrm{UCP} 2$ is proposed to control the speed of the TCA cycle, and production of mitochondrial ROS, and to promote the metabolic shift from glucose to fatty acid oxidation. ${ }^{48}$ OCTN2 transports long-chain fatty acids conjugated to carnitine into mitochondria for $\beta$-oxidation. $\beta$-oxidation is particularly implicated in CD pathogenesis, and consequently, pharmacological inhibition of intestinal fatty acid $\beta$-oxidation as well as genetic ablation of OCTN2 results in experimental colitis. ${ }^{49} 50$ These genetic risk factors might act in concert with other CD-relevant pathways described above and might render IECs particularly sensitive to environmental triggers of inflammation.

In patients with $\mathrm{CD}$, the proportion of PCs with aberrant granule structure and diffuse LYZ distribution has been shown to correlate with the cumulative number of NOD2 and ATG16L1 risk alleles, ${ }^{13}$ linking multiple CD genetic susceptibility loci to a defined PC phenotype. High proportions of abnormal PCs were associated with shorter time to disease recurrence after surgery in patients with a more aggressive clinical disease course. ${ }^{13}$ Our findings on PC granularity corroborate these findings in an independent patient cohort refining the endpoint of prediction to early postoperative lesions observed by ileocolonoscopy 6-12 months after surgery, and expand the set of predictive cellular markers to $\operatorname{Lgr} 5^{+}$ISC and to the location of expression. Abnormal PC morphology has also been linked to an activated immune response gene signature in crypts from patients with $\mathrm{CD}^{13}$ and to a transcriptional profile of cytokine stimulation in mice with hypomorphic expression of Atg1611. ${ }^{14}$ Together with the evidence presented here, this suggests an interrelated role of molecular inflammation and metabolism for ISC and PC phenotype. Furthermore, our data indicate some kind of signal propagation from adjacent, inflamed tissue regions, as jejunal organoids derived from inflamed $\mathrm{TNF}^{\triangle A R E}$ mice display impaired growth.

In conclusion, we identified ISC niche alterations as target of molecular inflammatory changes and predictive marker of early endoscopic recurrence in CD. In this context, we demonstrated that impaired mitochondrial function is linked to CD-associated loss of stemness and the generation of dysfunctional PC phenotypes. Mitochondria-derived signals might collaborate with IBD susceptibility genes to impact on ISC niche functionality, and in concert with environmental factors, such as intestinal microbiota or diet, contribute to the loss of ISC niche homeostasis observed in ileal CD. By implementing a drug-related shift towards mitochondrial respiration, we provide a proof-of-concept for the importance of mitochondrial metabolism in regulating ISC and PC functions and thereby rationalise a novel treatment approach for CD.

\section{Author affiliations}

${ }^{1}$ Chair of Nutrition and Immunology, Technische Universität München, FreisingWeihenstephan, Germany
²Department of Gastroenterology, Hôpital Saint-Louis, APHP, INSERM U1160, Université de Paris 1, Paris, Île-de-France, France

${ }^{3}$ Research Unit Analytical Pathology, Helmholtz Zentrum München, Neuherberg, Germany

${ }^{4}$ Chair of Nutrition Physiology, Technische Universität München, FreisingWeihenstephan, Germany

${ }^{5}$ ZIEL Institute for Food \& Health, Technische Universität München, München, Germany

Contributors SK, ER and DH designed the experiments, performed data analysis and wrote the manuscript. SK, ER and EB performed mouse and organoid culture experiments. SK performed tissue analysis of patients. AB performed the transmission electron microscopy. EG, NW, AM and PG supported analyses. MA and NH provided patient samples.

Funding DH received funding by the Deutsche Forschungsgemeinschaft (DFG, German Research Foundation) SFB 1371 (Projektnummer 395357507; P01) and Priority Programme SPP 1656. DH and MA received funding from the Helmsley Cheritable Trust (IBDOT).

Competing interests None declared.

Patient consent for publication Not required.

Provenance and peer review Not commissioned; externally peer reviewed.

Data availability statement All data relevant to the study are included in the article or uploaded as supplementary information. Additional data are available on request.

Open access This is an open access article distributed in accordance with the Creative Commons Attribution Non Commercial (CC BY-NC 4.0) license, which permits others to distribute, remix, adapt, build upon this work non-commercially, and license their derivative works on different terms, provided the original work is properly cited, appropriate credit is given, any changes made indicated, and the use is non-commercial. See: http://creativecommons.org/licenses/by-nc/4.0/.

\section{ORCID iDs}

Eva Rath http://orcid.org/0000-0003-1910-1162

Matthieu Allez http://orcid.org/0000-0002-2012-7522

Dirk Haller http://orcid.org/0000-0002-6977-4085

\section{REFERENCES}

1 Maloy KJ, Powrie F. Intestinal homeostasis and its breakdown in inflammatory bowel disease. Nature 2011;474:298-306.

2 Fritz T, Niederreiter L, Adolph T, et al. Crohn's disease: NOD2, autophagy and ER stress converge. Gut 2011;60:1580-8.

3 Jäger S, Stange EF, Wehkamp J. Inflammatory bowel disease: an impaired barrier disease. Langenbecks Arch Surg 2013;398:1-12.

4 Salzman NH, Ghosh D, Huttner KM, et al. Protection against enteric salmonellosis in transgenic mice expressing a human intestinal defensin. Nature 2003;422:522-6.

5 Salzman NH, Hung K, Haribhai D, et al. Enteric defensins are essential regulators of intestinal microbial ecology. Nat Immunol 2010;11:76-82.

6 Andersson-Rolf A, Zilbauer M, Koo B-K, et al. Stem cells in repair of gastrointestinal epithelia. Physiology 2017;32:278-89.

7 Yilmaz Ömer H., Katajisto P, Lamming DW, et al. mTORC1 in the Paneth cell niche couples intestinal stem-cell function to calorie intake. Nature 2012:486:490-5.

8 Rodríguez-Colman MJ, Schewe M, Meerlo M, et al. Interplay between metabolic identities in the intestinal crypt supports stem cell function. Nature 2017:543:424-7.

9 Schmitt M, Schewe M, Sacchetti A, et al. Paneth cells respond to inflammation and contribute to tissue regeneration by acquiring stem-like features through SCF/c-Kit signaling. Cell Rep 2018;24:2312-28

10 Roth S, Franken P, Sacchetti A, et al. Paneth cells in intestinal homeostasis and tissue injury. PLoS One 2012;7:e38965.

11 Yu S, Tong K, Zhao Y, et al. Paneth cell multipotency induced by Notch activation following injury. Cell Stem Cell 2018;23:46-59.

12 Kaser A, Lee A-H, Franke A, et al. XBP1 links ER stress to intestinal inflammation and confers genetic risk for human inflammatory bowel disease. Cell 2008;134:743-56.

13 VanDussen KL, Liu T-C, Li D, et al. Genetic variants synthesize to produce Paneth cell phenotypes that define subtypes of Crohn's disease. Gastroenterology 2014;146:200-9

14 Cadwell K, Liu JY, Brown SL, et al. A key role for autophagy and the autophagy gene Atg16l1 in mouse and human intestinal Paneth cells. Nature 2008;456:259-63.

15 Adolph TE, Tomczak MF, Niederreiter L, et al. Paneth cells as a site of origin for intestinal inflammation. Nature 2013;503:272-6.

16 Koslowski MJ, Kübler I, Chamaillard M, et al. Genetic variants of Wnt transcription factor TCF-4 (TCF7L2) putative promoter region are associated with small intestinal Crohn's disease. PLoS One 2009;4:e4496.

17 Günther C, Martini E, Wittkopf N, et al. Caspase-8 regulates TNF- $\alpha$-induced epithelial necroptosis and terminal ileitis. Nature 2011;477:335-9. 
18 Garabedian EM, Roberts LJ, McNevin MS, et al. Examining the role of Paneth cells in the small intestine by lineage ablation in transgenic mice. J Biol Chem 1997;272:23729-40.

19 Durand A, Donahue B, Peignon G, et al. Functional intestinal stem cells after Paneth cell ablation induced by the loss of transcription factor Math1 (Atoh1). Proc Natl Acad Sci U S A 2012;109:8965-70.

20 Schaubeck M, Clavel T, Calasan J, et al. Dysbiotic gut microbiota causes transmissible Crohn's disease-like ileitis independent of failure in antimicrobial defence. Gut 2016;65:225-37.

21 Rath E, Haller D. Mitochondria at the interface between danger signaling and metabolism: role of unfolded protein responses in chronic inflammation. Inflamm Bowel Dis 2012;18:1364-77.

22 Beltrán B, Nos P, Dasí F, et al. Mitochondrial dysfunction, persistent oxidative damage, and catalase inhibition in immune cells of naïve and treated Crohn's disease. Inflamm Bowel Dis 2010;16:76-86.

23 Fukushima K, Fiocchi C. Paradoxical decrease of mitochondrial DNA deletions in epithelial cells of active ulcerative colitis patients. Am J Physio/ Gastrointest Liver Physiol 2004;286:G804-13.

24 Rath E, Moschetta A, Haller D. Mitochondrial function - gatekeeper of intestinal epithelial cell homeostasis. Nat Rev Gastroenterol Hepatol 2018;15:497-516.

25 Rath E, Berger E, Messlik A, et al. Induction of dsRNA-activated protein kinase links mitochondrial unfolded protein response to the pathogenesis of intestinal inflammation. Gut 2012;61:1269-78.

26 Berger E, Rath E, Yuan D, et al. Mitochondrial function controls intestinal epithelial stemness and proliferation. Nat Commun 2016;7:13171.

27 Gassler N. Paneth cells in intestinal physiology and pathophysiology. World J Gastrointest Pathophysio/ 2017;8:150-60.

28 Auzolle C, Nancey S, Tran-Minh M-L, et al. Male gender, active smoking and previous intestinal resection are risk factors for post-operative endoscopic recurrence in Crohn's disease: results from a prospective cohort study. Aliment Pharmacol Ther 2018;48:924-32.

29 Gionchetti P, Dignass A, Danese S, et al. 3rd European Evidence-based Consensus on the Diagnosis and Management of Crohn's Disease 2016: Part 2: Surgical Management and Special Situations. J Crohns Colitis 2017;11:135-49.

30 Zhao Q, Wang J, Levichkin IV SS. A mitochondrial specific stress response in mammalian cells. EMBO J 2002:21:4411-9.

31 Takeda N, Jain R, LeBoeuf MR, et al. Interconversion between intestinal stem cell populations in distinct niches. Science 2011;334:1420-4.

32 Tian $\mathrm{H}$, Biehs $\mathrm{B}$, Warming $\mathrm{S}$, et al. A reserve stem cell population in small intestine renders LGR5-positive cells dispensable. Nature 2011:478:255-9.
33 Zietek T, Rath E. Chapter 3 - Intestinal organoids: Mini-guts grown in the laboratory. In: Davies JA, Lawrence ML, eds. Organs and organoids. Academic Press, 2018: 43-71.

34 Sato T, van Es JH, Snippert HJ, et al. Paneth cells constitute the niche for LGR5 stem cells in intestinal crypts. Nature 2011;469:415-8.

35 Kankotia S, Stacpoole PW. Dichloroacetate and cancer: new home for an orphan drug? Biochim Biophys Acta 2014;1846:617-29.

$36 \mathrm{Ng} \mathrm{SC}$, Shi HY, Hamidi N, et al. Worldwide incidence and prevalence of inflammatory bowel disease in the 21st century: a systematic review of population-based studies. Lancet 2018;390:2769-78.

37 Danese S. New therapies for inflammatory bowel disease: from the bench to the bedside. Gut 2012;61:918-32.

38 Bel S, Pendse M, Wang Y, et al. Paneth cells secrete lysozyme via secretory autophagy during bacterial infection of the intestine. Science 2017;357:1047-52.

39 Yan KS, Chia LA, Li X, et al. The intestinal stem cell markers BMI1 and LGR5 identify two functionally distinct populations. Proc Natl Acad Sci U S A 2012;109:466-71.

40 Giatromanolaki Aet al. Hypoxia inducible factor 1 and 2 overexpression in inflammatory bowel disease. J Clin Pathol 2003;56:209-13.

41 Donohoe DR, Garge N, Zhang X, et al. The microbiome and butyrate regulate energy metabolism and autophagy in the mammalian colon. Cell Metab 2011;13:517-26.

42 Waldschmitt N, Berger E, Rath E, et al. C/EBP homologous protein inhibits tissue repair in response to gut injury and is inversely regulated with chronic inflammation. Mucosal Immunol 2014;7:1452-66.

43 Soderholm JDet al. Augmented increase in tight junction permeability by luminal stimuli in the non-inflamed ileum of Crohn's disease. Gut 2002;50:307-13.

44 Colgan SP, Taylor CT. Hypoxia: an alarm signal during intestinal inflammation. Nat Rev Gastroenterol Hepatol 2010;7:281-7.

45 Colgan SP, Curtis VF, Campbell EL. The inflammatory tissue microenvironment in IBD. Inflamm Bowel Dis 2013;19:2238-44.

$46 \mathrm{Yu}$ X, Wieczorek S, Franke A, et al. Association of UCP2 -866 G/A polymorphism with chronic inflammatory diseases. Genes Immun 2009;10:601-5.

47 Barrett JC, Hansoul S, Nicolae DL, et al. Genome-Wide association defines more than 30 distinct susceptibility loci for Crohn's disease. Nat Genet 2008;40:955-62.

48 Pecqueur C, Bui T, Gelly C, et al. Uncoupling protein-2 controls proliferation by promoting fatty acid oxidation and limiting glycolysis-derived pyruvate utilization. FASEB J 2008:22:9-18.

49 Shekhawat PS, Srinivas SR, Matern D, et al. Spontaneous development of intestinal and colonic atrophy and inflammation in the carnitine-deficient JVS (OCTN2-/-) mice. Mol Genet Metab 2007;92:315-24.

50 Roediger WE, Nance $S$. Metabolic induction of experimental ulcerative colitis by inhibition of fatty acid oxidation. Br J Exp Pathol 1986;67:773-82. 